\title{
Positive Solutions for Boundary Value Problems of Fractional Differential Equation with Integral Boundary Conditions
}

\author{
Qiao Sun, ${ }^{1}$ Hongwei Ji, ${ }^{2}$ and Yujun Cui $\mathbb{D D}^{3}$ \\ ${ }^{1}$ Department of Applied Mathematics, Shandong University of Science and Technology, Qingdao 266590, China \\ ${ }^{2}$ Department of Mathematics and Physics, Nantong Normal College, Nantong 226010, China \\ ${ }^{3}$ State Key Laboratory of Mining Disaster Prevention and Control Co-Founded by Shandong Province and the Ministry of \\ Science and Technology, Shandong University of Science and Technology, Qingdao 266590, China
}

Correspondence should be addressed to Yujun Cui; cyj720201@163.com

Received 29 November 2017; Accepted 22 February 2018; Published 22 April 2018

Academic Editor: Dhananjay Gopal

Copyright (C) 2018 Qiao Sun et al. This is an open access article distributed under the Creative Commons Attribution License, which permits unrestricted use, distribution, and reproduction in any medium, provided the original work is properly cited.

By using two fixed-point theorems on cone, we discuss the existence results of positive solutions for the following boundary value problem of fractional differential equation with integral boundary conditions: $D_{0^{+}}^{\alpha} x(t)+a(t) f(t, x(t))=0, t \in(0,1), x(0)=x^{\prime}(0)=$ 0 , and $x(1)=\int_{0}^{1} x(t) d A(t)$.

\section{Introduction}

Boundary value problem for fractional differential equation has aroused much attention in the past few years; many professors devoted themselves to the solvability of fractional differential equations, especially to the study of the existence of solutions for boundary value problems of fractional differential equation (see [1-28]). For example, Wang et al. [19] studied the existence of positive solutions for the following problem:

$$
D_{0^{+}}^{\alpha} u(t)+\lambda f(t, u(t))=0, \quad t \in(0,1),
$$

with the boundary conditions

$$
u(0)=u^{\prime}(0)=u(1)=0
$$

where $D_{0^{+}}^{\alpha}$ is the Riemann-Liouville differential operator of $2<\alpha \leq 3, \lambda$ is a positive parameter, and $f$ may be singular at $t=0,1$ and may change sign. And Ma [14] discussed the positive solutions of

$$
D_{0^{+}}^{\alpha} u(t)+a(t) f(t, u(t))=0, \quad t \in(0,1),
$$

$$
\begin{aligned}
& u(0)=u^{\prime}(0)=0, \\
& u(1)=\sum_{i=1}^{m} \beta_{i} u\left(\xi_{i}\right),
\end{aligned}
$$

where $m \geq 1$ is integer and $\xi_{i}, \beta_{i}>0$.

There have already been lots of books and papers involving the positive solutions for boundary value problems of fractional differential equation; however, only a few papers cover that for fractional differential equation boundary value problems with integral boundary conditions. Motivated by [14], we shall investigate the positive solutions of the following boundary value problem:

$$
\begin{aligned}
D_{0^{+}}^{\alpha} x(t)+a(t) f(t, x(t)) & =0, \quad t \in(0,1), \\
x(0) & =x^{\prime}(0)=0, \\
x(1) & =\int_{0}^{1} x(t) d A(t),
\end{aligned}
$$

where $D_{0^{+}}^{\alpha}$ is the Riemann-Liouville differential operator of $2<\alpha<3, A(t)$ is right continuous on $[0,1)$, left continuous at $t=1$, and nondecreasing on $[0,1]$ with $A(0)=0$, and 
$\int_{0}^{1} u(t) d A(t)$ denotes the Riemann-Stieltjes integrals of $u$ with respect to $A$. And $a(t), f(t, x(t))$ satisfies the following conditions:

(H1) $a \in L[0,1]$ is nonnegative and not identically zero on any compact subset of $(0,1), \sigma=\int_{0}^{1} t^{\alpha-1} d A(t)<1$.

(H2) $f:[0,1] \times[0,+\infty) \rightarrow[0,+\infty)$ is continuous.

This paper consists of four sections. After the introduction, we recall some definitions, lemmas, and theorems in Section 2. And the main results of this paper are stated in Section 3. In the last section, we give two examples of the main results.

\section{Preliminaries}

Firstly, for convenience we recall some definitions, lemmas, and theorems.

Definition 1 (see $[29,30])$. Let $f \in L^{1}\left(R^{+}\right)$define the Riemann-Liouville fractional integral of order $\alpha>0$ for $f$ as

$$
I_{0^{+}}^{\alpha} f(t)=\frac{1}{\Gamma(\alpha)} \int_{0}^{t} f(s)(t-s)^{\alpha-1} d s,
$$

where $\Gamma(\alpha)$ is Euler gamma function.

Definition 2 (see $[29,30]$ ). Define the Riemann-Liouville fractional derivative of order $\alpha>0$ for $f$ as

$$
\begin{array}{r}
D_{0^{+}}^{\alpha} f(t)=\frac{1}{\Gamma(n-\alpha)}\left(\frac{d}{d t}\right)^{(n)} \int_{0}^{t} \frac{f(s)}{(t-s)^{\alpha+1-n}} d s, \\
n=[\alpha]+1,
\end{array}
$$

where $f$ has absolutely continuous derivatives up to order $(n-$ 1) on $R^{+}$.

Lemma 3. Let $y \in C[0,1], 2<\alpha \leq 3$; then the boundary value problem

$$
\begin{aligned}
D_{0^{+}}^{\alpha} x(t)+y(t) & =0, \quad t \in(0,1), \\
x(0) & =x^{\prime}(0)=0, \\
x(1) & =\int_{0}^{1} x(t) d A(t)
\end{aligned}
$$

has the unique solution $x(t)=\int_{0}^{1} G_{1}(t, s) y(s) d s$, where

$$
\begin{aligned}
& G_{1}(t, s)=G(t, s)+\frac{t^{\alpha-1}}{1-\sigma} \int_{0}^{1} G(\tau, s) d A(\tau), \\
& G(t, s) \\
& \quad= \begin{cases}\frac{t^{\alpha-1}(1-s)^{\alpha-1}-(t-s)^{\alpha-1}}{\Gamma(\alpha)}, & 0 \leq s \leq t \leq 1 ; \\
\frac{t^{\alpha-1}(1-s)^{\alpha-1}}{\Gamma(\alpha)}, & 0 \leq t \leq s \leq 1 .\end{cases}
\end{aligned}
$$

Proof. The boundary value problem can be converted to an equivalent integral equation:

$$
\begin{array}{r}
x(t)=-I_{0^{+}}^{\alpha} y(t)+c_{1} t^{\alpha-1}+c_{2} t^{\alpha-2}+c_{3} t^{\alpha-3}, \\
\\
c_{1}, c_{2}, c_{3} \in R .
\end{array}
$$

Then the solution is

$$
\begin{aligned}
x(t)= & -\frac{1}{\Gamma(\alpha)} \int_{0}^{t} y(s)(t-s)^{\alpha-1} d s+c_{1} t^{\alpha-1}+c_{2} t^{\alpha-2} \\
& +c_{3} t^{\alpha-3}
\end{aligned}
$$

It follows from the boundary conditions $x(0)=x^{\prime}(0)=0$ that $c_{3}=c_{2}=0$ and

$$
c_{1}=\frac{1}{\Gamma(\alpha)} \int_{0}^{1} y(s)(1-s)^{\alpha-1} d s+\int_{0}^{1} x(s) d A(s) .
$$

Thus we get

$$
\begin{aligned}
x(t)= & -\frac{1}{\Gamma(\alpha)} \int_{0}^{t} y(s)(t-s)^{\alpha-1} d s \\
& +\frac{1}{\Gamma(\alpha)} \int_{0}^{1} y(s)(1-s)^{\alpha-1} d s \cdot t^{\alpha-1} \\
& +\int_{0}^{1} x(s) d A(s) \cdot t^{\alpha-1} \\
= & \int_{0}^{1} G(t, s) y(s) d s+\int_{0}^{1} x(s) d A(s) \cdot t^{\alpha-1}
\end{aligned}
$$

Then we can obtain

$$
\begin{aligned}
& \int_{0}^{1} x(s) d A(s)=\int_{0}^{1}\left[\int_{0}^{1} G(s, \tau) y(\tau) d \tau\right. \\
& \left.+\int_{0}^{1} x(\tau) d A(\tau) s^{\alpha-1}\right] d A(s) \\
& \quad=\int_{0}^{1} \int_{0}^{1} G(s, \tau) y(\tau) d \tau d A(s) \\
& +\int_{0}^{1} x(s) d A(s) \cdot \int_{0}^{1} t^{\alpha-1} d A(t)
\end{aligned}
$$

which means

$$
\int_{0}^{1} x(s) d A(s)=\frac{\int_{0}^{1} \int_{0}^{1} G(s, \tau) y(\tau) d \tau d A(s)}{1-\int_{0}^{1} t^{\alpha-1} d A(t)} .
$$


So

$x(t)$

$$
\begin{aligned}
= & \int_{0}^{1} G(t, s) y(s) d s \\
& +\frac{\int_{0}^{1} \int_{0}^{1} G(s, \tau) y(\tau) d \tau d A(s)}{1-\int_{0}^{1} t^{\alpha-1} d A(s)} t^{\alpha-1} \\
= & \int_{0}^{1}\left[G(t, s)+\frac{t^{\alpha-1}}{1-\sigma} \int_{0}^{1} G(\tau, s) d A(\tau)\right] y(s) d s \\
= & \int_{0}^{1} G_{1}(t, s) y(s) d s .
\end{aligned}
$$

Lemma 4 (see [20]). $G(t, s)$ defined in (9) has the following properties:

(i) $G(t, s)>0, t, s \in(0,1)$.

(ii) $G(t, s)=G(1-s, 1-t), t, s \in[0,1]$.

(iii) $k(1-t) k(s) \leq \Gamma(\alpha) G(t, s) \leq(\alpha-1) k(s), t, s \in[0,1]$ where $k(t)=t(1-t)^{\alpha-1}$.

Lemma 5. If (H2) is satisfied, then $G_{1}(t, s)$ defined in (8) has the following properties:

$$
k(1-t) k(s) \leq \Gamma(\alpha) G_{1}(t, s) \leq L k(s), \quad t, s \in[0,1],
$$

where $L=(\alpha-1)\left(1+\int_{0}^{1} d A(s) /(1-\sigma)\right)$.

Proof. The proof can be easily accomplished by Lemma 4, so we omitted it.

Theorem 6 (see [31]). Let $E$ be a Banach space and $P \subseteq E$ be a cone in E. Suppose that $\Omega_{1}, \Omega_{2}$ are two bounded open sets of $E$ with $\overline{\Omega_{1}} \subset \Omega_{2}$. Assume that $T: P \cap\left(\overline{\Omega_{2}} \backslash \Omega_{1}\right) \rightarrow P$ is a completely continuous operator such that either

(i) $\|T x\| \leq\|x\|$ for any $x \in P \cap \partial \Omega_{1}$ and $\|T x\| \geq\|x\|$ for any $x \in P \cap \partial \Omega_{2}$ or

(ii) $\|T x\| \geq\|x\|$ for any $x \in P \cap \partial \Omega_{1}$ and $\|T x\| \leq\|x\|$ for any $x \in P \cap \partial \Omega_{2}$.

Then $T$ has a fixed point in $P \cap\left(\overline{\Omega_{2}} \backslash \Omega_{1}\right)$.

Theorem 7 (see [32]). Let $P$ be a cone of a Banach space E. $P_{c}=\{x \in P:\|x\|<c\} . \theta$ is a nonnegative continuous concave function on $P$, such that, for any $x \in \overline{P_{c}}, \theta(x) \leq\|x\|$, and $P(\theta, b, d)=\{x \in P: \theta(x) \geq b,\|x\| \leq d\}$. Assume that $T:$ $\overline{P_{c}} \rightarrow \overline{P_{c}}$ is completely continuous, and there exist constants $a<b<d \leq c$ such that

(c1) $\{x \in P(\theta, b, d): \theta(x)>b\} \neq \emptyset$ and $\theta(T x)>b$ for $x \in P(\theta, b, d)$;

(c2) $\|T x\|<a$ for $x \in \overline{P_{a}}$;

(c3) $\theta(T x)>b$ for any $x \in P(\theta, b, c)$ with $\|T x\|>d$.
Then $T$ has at least three fixed points $x_{1}, x_{2}, x_{3}$ with $\left\|x_{1}\right\|<a$, $b<\theta\left(x_{2}\right),\left\|x_{3}\right\|>a$, and $\theta\left(x_{3}\right)<b$.

Let $E=C[0,1]$ be a Banach space with the maximum norm $\|x\|=\max _{0 \leq t \leq 1}|x(t)|$; define the cone $P \subseteq E$ as

$$
P=\{x \in E: x(t) \geq 0, t \in[0,1]\} .
$$

Define a continuous operator $T: P \rightarrow E$ as

$$
(T x)(t)=\int_{0}^{1} G_{1}(t, s) a(s) f(s, x(s)) d s .
$$

Lemma 8. Assume that (H1) and (H2) hold; then $T: P \rightarrow P$ is a completely continuous operator.

Proof. The lemma can be easily proven, so we omitted it.

\section{Main Results}

We define the following notation: given $\delta \in(0,1 / 2)$, take

$$
\begin{aligned}
h & =\min _{\delta \leq t \leq 1-\delta} k(1-t)=\min _{\delta \leq t \leq 1-\delta}(1-t) t^{\alpha-1}, \\
M & =\frac{\Gamma(\alpha)}{L \int_{0}^{1} k(s) a(s) d s}, \\
N & =\frac{\Gamma(\alpha)}{h \int_{\delta}^{1-\delta} k(s) a(s) d s} .
\end{aligned}
$$

Now we can obtain the following theorems.

Theorem 9. Suppose that (H1) and (H2) are satisfied; there exist two positive constants $r_{2}>r_{1}>0$ such that

(H3) $f(t, x) \leq M r_{2},(t, x) \in[0,1] \times\left[0, r_{2}\right]$;

(H4) $f(t, x) \geq N r_{1},(t, x) \in[0,1] \times\left[0, r_{1}\right]$.

Then boundary value problem (4) has at least one positive solution $x \in P$ such that $r_{1} \leq\|x\| \leq r_{2}$.

Proof. The solution of boundary value problem (4) is equivalent to the fixed point of operator $T$. Let $\Omega_{2}=\{x \in P:\|x\|<$ $\left.r_{2}\right\}$; when $x \in \partial \Omega_{2}$, for any $t \in[0,1]$ we have $0 \leq x(t) \leq r_{2}$. By Lemma 5 and (H3) we get

$$
\begin{aligned}
(T x)(t) & =\int_{0}^{1} G_{1}(t, s) a(s) f(s, x(s)) d s \\
& \leq \frac{L M r_{2}}{\Gamma(\alpha)} \int_{0}^{1} k(s) a(s) d s=r_{2}=\|x\|,
\end{aligned}
$$

which means when $x \in \partial \Omega_{2},\|T x\| \leq\|x\|$. 
Let $\Omega_{1}=\left\{x \in P:\|x\|<r_{1}\right\}$; when $x \in \partial \Omega_{1}$, for any $t \in[0,1]$ we have $0 \leq x(t) \leq r_{1}$. By Lemma 5 and (H4) we get

$$
\begin{aligned}
(T x)(t) & =\int_{0}^{1} G_{1}(t, s) a(s) f(s, x(s)) d s \\
& \geq k(1-t) \frac{N r_{1}}{\Gamma(\alpha)} \int_{0}^{1} k(s) a(s) d s \\
& \geq \min _{\delta \leq t \leq 1-\delta} k(1-t) \frac{N r_{1}}{\Gamma(\alpha)} \int_{\delta}^{1-\delta} k(s) a(s) d s \\
& =r_{1}=\|x\|,
\end{aligned}
$$

which means when $x \in \partial \Omega_{1},\|T x\| \geq\|x\|$.

It follows from Theorem 6 that we know that $T$ has at least one fixed point in $\left(\overline{\Omega_{2}} \backslash \Omega_{1}\right)$, which means that the boundary value problem (4) has at least one solution.

Theorem 10. Suppose that (H1) and (H2) are satisfied; there exist four positive constants $a, b, c, d$ with $0<a<b<$ $(h / L) d<d<c$, such that

(H5) $f(t, x)<M a,(t, x) \in[0,1] \times[0, a]$;

(H6) $f(t, x)>N b,(t, x) \in[\delta, 1-\delta] \times[b, d]$;

(H7) $f(t, x) \leq M c,(t, x) \in[0,1] \times[0, c]$.

Then boundary value problem (4) has at least three positive solutions $x_{1}, x_{2}, x_{3}$, such that

$$
\begin{aligned}
\max _{0 \leq t \leq 1}\left|x_{1}(t)\right| & <a, \\
b & <\min _{\delta \leq t \leq 1-\delta}\left|x_{2}(t)\right|<\max _{0 \leq t \leq 1}\left|x_{2}(t)\right| \leq c, \\
a & <\max _{0 \leq t \leq 1}\left|x_{3}(t)\right| \leq c, \\
\min _{\delta \leq t \leq 1-\delta}\left|x_{3}(t)\right| & <b .
\end{aligned}
$$

Proof. Define a nonnegative continuous concave function $\theta$ on $P$ as

$$
\theta(x)=\min _{\delta \leq t \leq 1-\delta} x(t)
$$

If $x \in \overline{P_{c}}=\{x \in P:\|x\| \leq c\}$, then $\|x\| \leq c$; it follows from (H7) that $f(t, x) \leq M c$; hence

$$
\begin{aligned}
\|T x\| & \leq \frac{L}{\Gamma(\alpha)} \int_{0}^{1} k(s) a(s) f(s, x(s)) d s \\
& \leq \frac{L M c}{\Gamma(\alpha)} \int_{0}^{1} k(s) a(s) d s=c .
\end{aligned}
$$

Thus, $T\left(\overline{P_{c}}\right) \subseteq \overline{P_{c}}$. It follows from Lemma 8 that $T$ is completely continuous. In the same way, let $x \in \overline{P_{a}}$; it follows from (H5) that $f(t, x)<M a$ for any $t \in[0,1]$, which shows that condition (c2) of Theorem 7 is fulfilled.

Let $x(t)=(b+d) / 2$; it is easy to know that $x \in P(\theta, b, d)$ and $\{x \in P(\theta, b, d): \theta(x)>b\} \neq \emptyset$. If $x \in P(\theta, b, d)$, we have $b \leq x(t) \leq d$ for any $t \in[\delta, 1-\delta]$. We know $f(t, x(t))>N b$ for $\delta \leq t \leq 1-\delta$ by (H6). So we get

$$
\begin{aligned}
\theta(T x) & =\min _{\delta \leq t \leq 1-\delta} T x(t) \\
& =\min _{\delta \leq t \leq 1-\delta} \int_{0}^{1} G_{1}(t, s) a(s) f(s, x(s)) d s \\
& \geq \min _{\delta \leq t \leq 1-\delta} \int_{0}^{1} \frac{k(1-t) k(s)}{\Gamma(\alpha)} a(s) f(s, x(s)) d s \\
& >\frac{N h b}{\Gamma(\alpha)} \int_{\delta}^{1-\delta} k(s) a(s) d s=b .
\end{aligned}
$$

So condition (c1) of Theorem 7 holds.

When $x \in P(\theta, b, c)$ with $\|T x\|>d$, noting that

$$
\|T x\| \leq \frac{L}{\Gamma(\alpha)} \int_{0}^{1} k(s) a(s) f(s, x(s)) d s,
$$

thus

$$
\begin{aligned}
(T x)(t) & \geq \frac{k(1-t)}{\Gamma(\alpha)} \int_{0}^{1} k(s) a(s) f(s, x(s)) d s \\
& \geq \frac{k(1-t)}{L}\|T x\|,
\end{aligned}
$$

so we obtain

$$
\begin{aligned}
\theta(T x) & =\min _{\delta \leq t \leq 1-\delta} T x(t) \geq \frac{\min _{\delta \leq t \leq 1-\delta} k(1-t)}{L}\|T x\| \\
& >\frac{h}{L} d>b .
\end{aligned}
$$

That is to say, (c3) is satisfied.

All conditions of Theorem 7 are satisfied, so $T$ has at least three fixed points $x_{1}, x_{2}, x_{3}$, which means that the boundary value problem (4) has at least three positive solutions $x_{1}, x_{2}$, $x_{3}$, such that

$$
\begin{aligned}
\max _{0 \leq t \leq 1}\left|x_{1}(t)\right| & <a, \\
b & <\min _{\delta \leq t \leq 1-\delta}\left|x_{2}(t)\right|<\max _{0 \leq t \leq 1}\left|x_{2}(t)\right| \leq c, \\
a & <\max _{0 \leq t \leq 1}\left|x_{3}(t)\right| \leq c, \\
\min _{\delta \leq t \leq 1-\delta}\left|x_{3}(t)\right| & <b .
\end{aligned}
$$

The proof of this theorem is finished.

\section{Some Examples}

Now we present two examples to illustrate our main results.

Example 1. Let us see the following problem:

$$
\begin{aligned}
D_{0^{+}}^{5 / 2} x(t)+\frac{1}{4} \sin t+x+3 & =0, \quad t \in(0,1), \\
x(0) & =x^{\prime}(0)=0, \\
x(1) & =\int_{0}^{1} x(t) d t .
\end{aligned}
$$


Choose $\delta=1 / 3$; we obtain that $L=4, h=2 / \sqrt{243}$, $M=105 \sqrt{\pi} / 64 \approx 2.91$, and $N=76545 \sqrt{\pi} /(704 \sqrt{2}-256) \approx$ 183.48. Then for any $(t, x) \in[0,1] \times[0,2]$, we have $f(t, x)=$ $(1 / 4) \sin t+x+3 \leq 5.25 \leq M r_{2} \approx 5.82$, and for any $(t, x) \in$ $[0,1] \times[0,0.01]$, we get $f(t, x)=(1 / 4) \sin t+x+3 \geq 3 \geq$ $N r_{1} \approx 1.83$.

Then the boundary value problem has at least one positive solution $x \in P$ such that $0.01 \leq\|x\| \leq 2$.

Example 2. We now study the following problem:

$$
\begin{aligned}
D_{0^{+}}^{5 / 2} x(t)+f(t, x) & =0, \quad t \in(0,1), \\
x(0) & =x^{\prime}(0)=0, \\
x(1) & =\int_{0}^{1} x(t) d t,
\end{aligned}
$$

where

$$
f(t, x)= \begin{cases}\frac{t}{3}+x^{2}, & x \leq 1 \\ 183+\frac{t}{3}+x, & x>1\end{cases}
$$

Choose $\delta=1 / 3$; we have $M \approx 2.91, N \approx 183.48, L=4$, and $h \approx 0.1283$. Let $a=1 / 2, b=1, c=100$, and $d=35$; then for any $(t, x) \in[0,1] \times[0,1 / 2]$, we have $f(t, x)=t / 3+x^{2} \leq$ $0.59<M a \approx 1.46$, for any $(t, x) \in[1 / 3,2 / 3] \times[1,35]$, we have $f(t, x)=183+t / 3+x \geq 184.11>N b \approx 183.48$, and for any $(t, x) \in[0,1] \times[0,100]$, we have $f(t, x)=183+t / 3+$ $x \leq 283.34<M c \approx 291$. Then by Theorem 10 , we conclude that this boundary value problem has at least three positive solutions $x_{1}, x_{2}, x_{3}$, such that

$$
\begin{aligned}
\max _{0 \leq t \leq 1}\left|x_{1}(t)\right| & <\frac{1}{2} \\
1 & <\min _{\delta \leq t \leq 1-\delta}\left|x_{2}(t)\right|<\max _{0 \leq t \leq 1}\left|x_{2}(t)\right| \leq 100 \\
\frac{1}{2} & <\max _{0 \leq t \leq 1}\left|x_{3}(t)\right| \leq 100 \\
\min _{\delta \leq t \leq 1-\delta}\left|x_{3}(t)\right| & <1 .
\end{aligned}
$$

\section{Conflicts of Interest}

The authors declare that they have no conflicts of interest.

\section{Acknowledgments}

This project is supported by NNSF of China (11371221, 11571207), SDNSF (ZR2018MA011), and the Tai'shan Scholar Engineering Construction Fund of Shandong Province of China.

\section{References}

[1] Z. Bai, "On positive solutions of a nonlocal fractional boundary value problem," Nonlinear Analysis: Theory, Methods \& Applications, vol. 72, no. 2, pp. 916-924, 2010.
[2] Y. Cui, "Uniqueness of solution for boundary value problems for fractional differential equations," Applied Mathematics Letters, vol. 51, pp. 48-54, 2016.

[3] Y. Cui, "Existence results for singular boundary value problem of nonlinear fractional differential equation," Abstract and Applied Analysis, vol. 2011, Article ID 605614, 2011.

[4] Y. Cui, Q. Sun, and X. Su, "Monotone iterative technique for nonlinear boundary value problems of fractional order $\mathrm{p} \in(2$, 3 ]," Advances in Difference Equations, vol. 2017, no. 1, article no. 248, 2017.

[5] Y. Cu, W. Ma, Q. Sun, and X. Su, "New uniqueness results for boundary value problem of fractional differential equation," Nonlinear Analysis: Modelling and Control, pp. 31-39, 2018.

[6] H. H. Dong, B. Y. Guo, and B. S. Yin, "Generalized fractional supertrace identity for Hamiltonian structure of NLS-MKdV hierarchy with self-consistent sources," Analysis and Mathematical Physics, vol. 6, no. 2, pp. 199-209, 2016.

[7] A. M. El-Sayed, "Nonlinear functional-differential equations of arbitrary orders," Nonlinear Analysis: Theory, Methods \& Applications, vol. 33, no. 2, pp. 181-186, 1998.

[8] J. R. Graef and L. Kong, "Positive solutions for a class of higher order boundary value problems with fractional $q$-derivatives," Applied Mathematics and Computation, vol. 218, no. 19, pp. 9682-9689, 2012.

[9] L. Guo, L. Liu, and Y. Wu, "Existence of positive solutions for singular fractional differential equations with infinite-point boundary conditions," Lithuanian Association of Nonlinear Analysts. Nonlinear Analysis: Modelling and Control, vol. 21, no. 5, pp. 635-650, 2016.

[10] H. Maagli, "Existence of positive solutions for a nonlinear fractional differential equation," Electronic Journal of Differential Equations, vol. 2013, 2013.

[11] D. Jiang and C. Yuan, "The positive properties of the Green function for Dirichlet-type boundary value problems of nonlinear fractional differential equations and its application," Nonlinear Analysis: Theory, Methods \& Applications, vol. 72, no. 2, pp. 710-719, 2010.

[12] L. Liu, H. Li, C. Liu, and Y. Wu, "Existence and uniqueness of positive solutions for singular fractional differential systems with coupled integral boundary conditions," Journal of Nonlinear Sciences and Applications. JNSA, vol. 10, no. 1, pp. 243-262, 2017.

[13] D. Liu, Z. Ouyang, and H. Wang, "Positive solutions for class of state dependent boundary value problems with fractional order differential operators," Abstract and Applied Analysis, vol. 2015, Article ID 263748, 2015.

[14] D.-x. Ma, "Positive solutions of multi-point boundary value problem of fractional differential equation," Arab Journal of Mathematical Sciences, vol. 21, no. 2, pp. 225-236, 2015.

[15] N. Nyamoradi, "Existence of solutions for multi point boundary value problems for fractional differential equations," Arab Journal of Mathematical Sciences, vol. 18, no. 2, pp. 165-175, 2012.

[16] T. Qi, Y. Liu, and Y. Cui, "Existence of Solutions for a Class of Coupled Fractional Differential Systems with Nonlocal Boundary Conditions," Journal of Function Spaces, vol. 2017, Article ID 6703860, 2017.

[17] T. Qi, Y. Liu, and Y. Zou, "Existence result for a class of coupled fractional differential systems with integral boundary value conditions," Journal of Nonlinear Sciences and Applications. JNSA, vol. 10, no. 7, pp. 4034-4045, 2017. 
[18] Y. Wang, L. Liu, X. Zhang, and Y. Wu, "Positive solutions of an abstract fractional semipositone differential system model for bioprocesses of HIV infection," Applied Mathematics and Computation, vol. 258, pp. 312-324, 2015.

[19] Y. Wang, L. Liu, and Y. Wu, "Positive solutions for a class of fractional boundary value problem with changing sign nonlinearity," Nonlinear Analysis: Theory, Methods \& Applications, vol. 74, no. 17, pp. 6434-6441, 2011.

[20] X. Zhang, L. Liu, and Y. Wu, "Multiple positive solutions of a singular fractional differential equation with negatively perturbed term," Mathematical and Computer Modelling, vol. 55, no. 3-4, pp. 1263-1274, 2012.

[21] X. Zhang, L. Liu, B. Wiwatanapataphee, and Y. Wu, "The eigenvalue for a class of singular $p$-Laplacian fractional differential equations involving the Riemann-Stieltjes integral boundary condition," Applied Mathematics and Computation, vol. 235, pp. 412-422, 2014.

[22] X. Zhang, L. Liu, and Y. Wu, "Variational structure and multiple solutions for a fractional advection-dispersion equation," Computers \& Mathematics with Applications. An International Journal, vol. 68, no. 12, part A, pp. 1794-1805, 2014.

[23] D. Zhao and Y. Liu, "Positive solutions for a class of fractional differential coupled system with integral boundary value conditions," Journal of Nonlinear Sciences and Applications. JNSA, vol. 9, no. 5, pp. 2922-2942, 2016.

[24] Y. Zhao, S. Sun, Z. Han, and Q. Li, "The existence of multiple positive solutions for boundary value problems of nonlinear fractional differential equations," Communications in Nonlinear Science and Numerical Simulation, vol. 16, no. 4, pp. 2086-2097, 2011.

[25] B. Zhu, L. Liu, and Y. Wu, "Local and global existence of mild solutions for a class of nonlinear fractional reaction-diffusion equations with delay," Applied Mathematics Letters, vol. 61, pp. 73-79, 2016.

[26] Y. Zou and G. He, "On the uniqueness of solutions for a class of fractional differential equations," Applied Mathematics Letters, vol. 74, pp. 68-73, 2017.

[27] Y. Zou and G. He, "The existence of solutions to integral boundary value problems of fractional differential equations at resonance," Journal of Function Spaces, Art. ID 2785937, 7 pages, 2017.

[28] Y. Zou and Y. Cui, "Existence results for a functional boundary value problem of fractional differential equations," Advances in Difference Equations, vol. 2013, article no. 233, 2013.

[29] A. A. Kilbas, H. M. Srivastava, and J. J. Trujillo, Theory and Applications of Fractional Differential Equations, New York, NY, USA, Elsevier, 2006.

[30] K. S. Miller and B. Ross, An Introduction to the Fractional Calculus and Fractional Differential Equations, A Wiley-Interscience Publication, John Wiley \& Sons, New York, NY, USA, 1993.

[31] M. A. Krasnoselskii, Positive Solutions of Operator Equations, Noordhoff, Groningen, The Netherlands, 1964.

[32] R. W. Leggett and L. R. Williams, "Multiple positive fixed points of nonlinear operators on ordered Banach spaces," Indiana University Mathematics Journal, vol. 28, no. 4, pp. 673-688, 1979. 


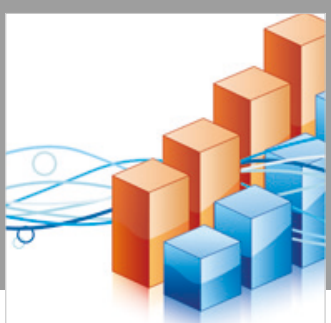

Advances in

Operations Research

\section{-n-m}
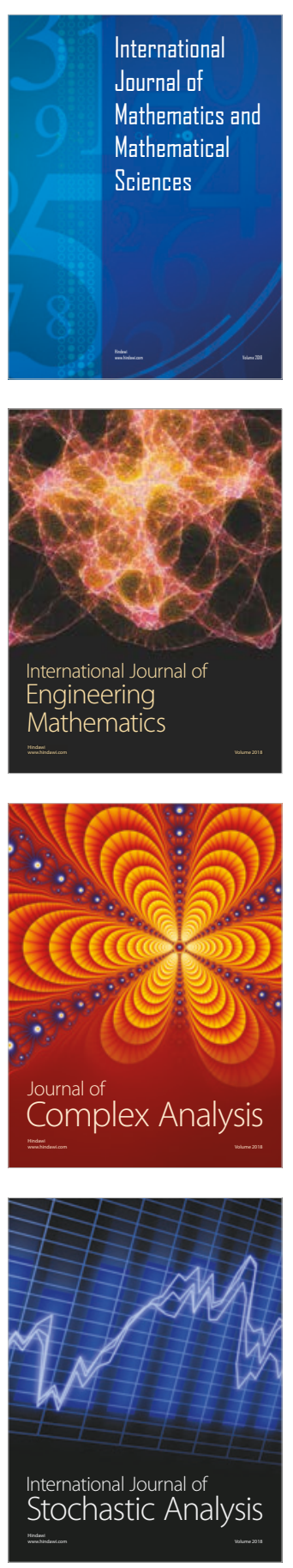
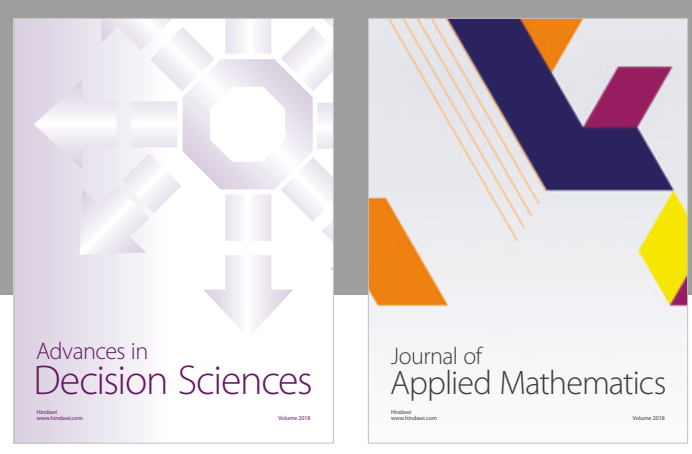

Journal of

Applied Mathematics
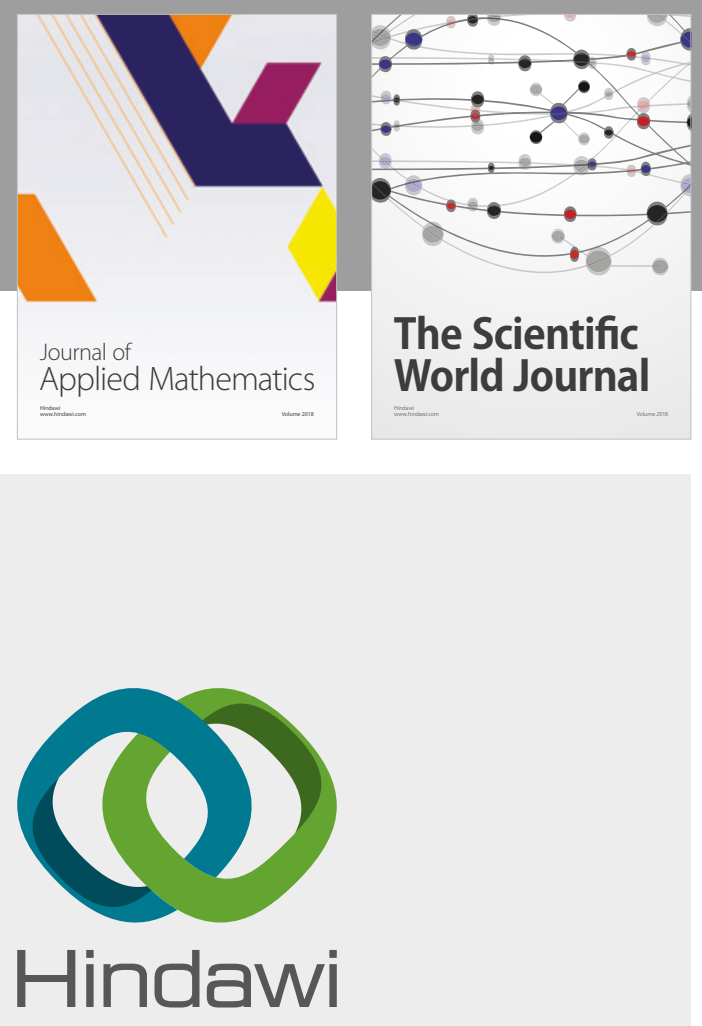

Submit your manuscripts at

www.hindawi.com

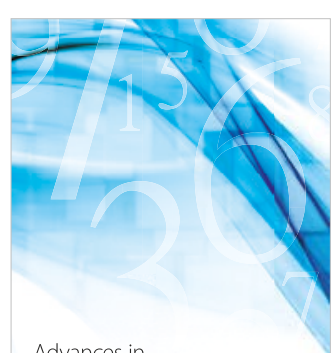

Advances in
Numerical Analysis
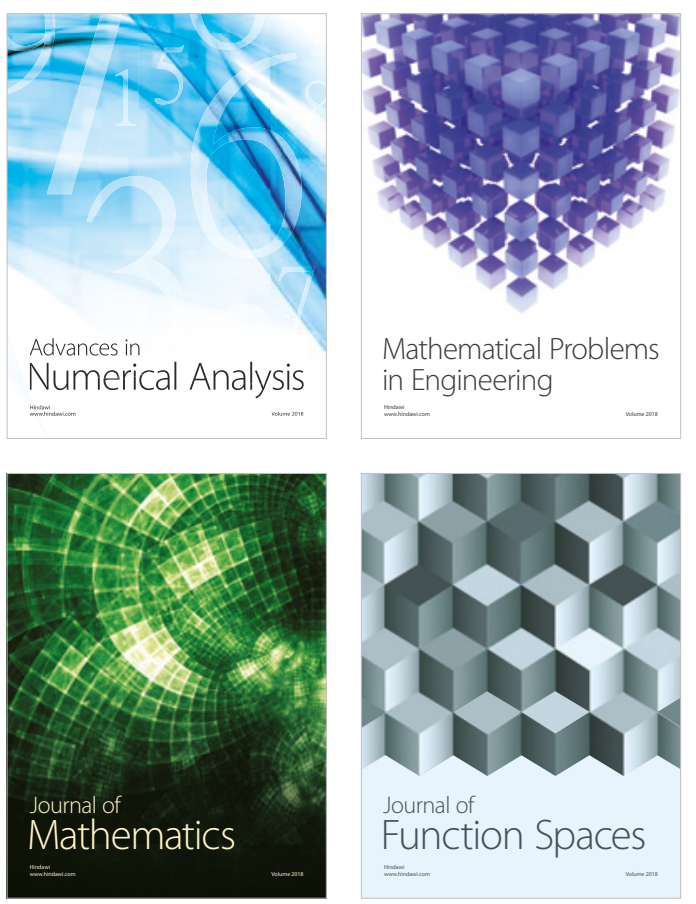

Mathematical Problems in Engineering

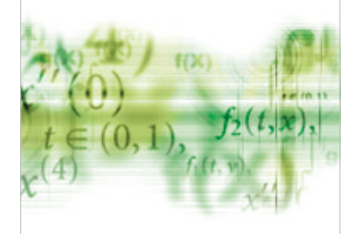

International Journal of

Differential Equations

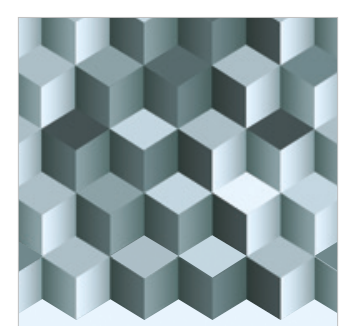

Journal of

Function Spaces

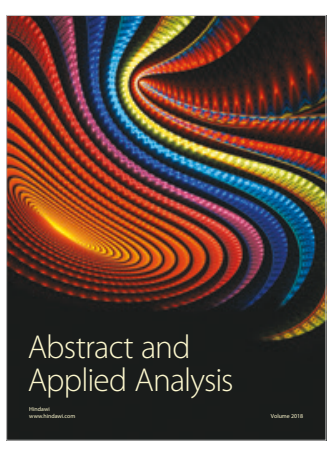

The Scientific

World Journal

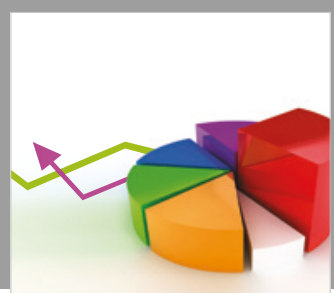

Journal of

Probability and Statistics
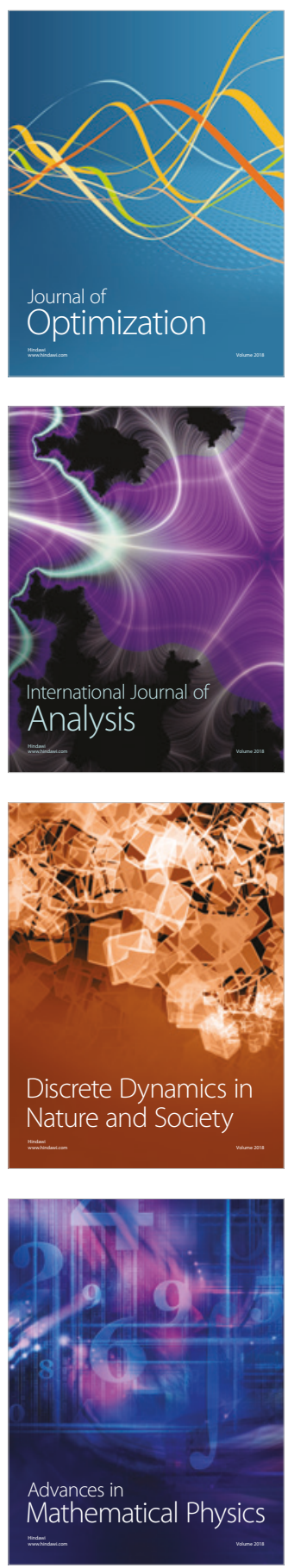